\title{
Non-equilibrium critical dynamics with domain wall and surface
}

\author{
N. J. Zhou ${ }^{1,2}$ and B. Zheng ${ }^{1, *}$ \\ 1 Zhejiang University, Zhejiang Institute of Modern Physics, Hangzhou 310027, P.R. China \\ 2 Department of Physics, National Central University, Chungli, Taiwan 320
}

\begin{abstract}
With Monte Carlo simulations, we investigate the relaxation dynamics with a domain wall for magnetic systems at the critical temperature. The dynamic scaling behavior is carefully analyzed, and a dynamic roughening process is observed. For comparison, similar analysis is applied to the relaxation dynamics with a free or disordered surface.
\end{abstract}

PACS numbers: $64.60 . \mathrm{Ht}, 68.35 . \mathrm{Rh}, 05.10 . \mathrm{Ln}$

\footnotetext{
* corresponding author: zheng@zimp.zju.edu.cn
} 


\section{INTRODUCTION}

In the past years, much effort of physicists has been devoted to the understanding of nonequilibrium dynamic processes. Phase ordering dynamics, spin glass dynamics, structural glass dynamics and interface growth etc are important examples. Since the pioneer work by Janssen et al [1], the universal dynamic scaling form in critical dynamics has been explored up to the macroscopic short-time regime [1-10], when the system is still far from equilibrium. Although the spatial correlation length is still short in the beginning of the time evolution, the short-time dynamic scaling form is induced by the divergent correlating time around a continuous phase transition. Based on the short-time dynamic scaling form, new methods for the determination of both dynamic and static critical exponents as well as the critical temperature have been developed [4, 5, 7 -11]. Since the measurements are carried out in the short-time regime, one does not suffer from critical slowing down.

In understanding the dynamic scaling form far from equilibrium, we should keep in mind that it holds after a time scale $t_{m i c}$, which is sufficiently long in the microscopic sense, but still short in the macroscopic sense. More importantly, the macroscopic initial condition should be taken into account in the dynamic scaling form [1, 9, 12]. For the dynamic relaxation starting from an ordered state, i.e., a state with an initial magnetization $m_{0}=1$, for example, the magnetization decays by a power law [8, 9, 12]. If $m_{0}$ is smaller but close to 1, there emerge corrections to scaling. For the dynamic relaxation starting from a random state, i.e., a state without spatial correlations and with a small $m_{0}$, however, the magnetization does not decay, and rather shows an initial increase in the macroscopic short-time regime. An independent critical exponent $x_{0}$ must be introduced to describe the scaling dimension of the initial magnetization [1, 6, 9, 10]. If $m_{0}=0$, the magnetization naturally remains zero during the dynamic evolution, but $x_{0}$ is still needed to describe the auto-correlation function etc. This critical exponent also explains the power-law decay of the remanent magnetization in spin glasses [2, 13, 14]. On the other hand, the short-time dynamic scaling form is universal, in the sense that it does not depend on the microscopic details of the dynamic system, such as the lattice types, interactions, and updating schemes etc. Up to now, the dynamic relaxation with the ordered and random initial states has been systematically investigated.

Recent progress in the non-equilibrium critical dynamics and its applications includes, for 
example, theoretical calculations and numerical simulations of the XY models and Josephson junction arrays [15 18], magnets with quenched disorder [19 23], and various critical systems [24-27]. Dynamic reweighting methods have been proposed [20, 28], and the dynamic approach to the weak first-order phase transitions is also attractive [20, 29 31]. Recently, the ageing phenomenon around a continuous phase transition has been also intensively studied [32 38]. In this case, the dynamic scaling form for the ageing phenomenon is induced by the long-range time correlation, different from that induced by meta-stable states in glassy systems below the transition temperature $T_{c}$.

On the other hand, in the past years many activities have been devoted to the domain-wall dynamics [39 45]. For magnetic materials, for example, a domain wall separates domains with different spin orientations. Microscopically, the domain wall may move and create bubbles, and macroscopically, it may propagate and roughen. At the zero temperature, there occurs a pinning-depinning phase transition induced by quenched randomness [39, 46, 47]. For a magnetic system with weak disorder, the domain wall does not propagate unless the external magnetic field $h$ exceeds a threshold $h_{c}$. At the critical field $h_{c}$, a roughening phenomenon is also observed [48]. When a periodic external field $h(t)=h_{0} \cos (\omega t)$ is applied, the second-order phase transition is softened to a hysteresis loop [39 41, 43]. Most these works concerning the domain-wall motion concentrate on the stationary state at the zero or very low temperatures and in response to the external magnetic field $h(t)$.

In this paper, we systematically investigate the dynamic relaxation with a domain wall at the critical temperature. For simplicity, we assume that no external field is applied. Macroscopically, therefore, the domain wall does not propagate. We should only keep in mind that different from the case at the zero temperature, here the bulk also evolves in time. To be specific, we consider the dynamic relaxation starting from a semi-ordered initial state. For the Ising model, for example, the semi-ordered initial state consists of two fullyordered domains with opposite spin orientations. As time evolves, the domain wall roughens, and looks like a growing interface. In this paper, we call it the domain interface. Such a domain-wall dynamics is theoretically and practically important. Theoretically, it is very interesting to investigate the short-time dynamic scaling behavior starting from the semiordered state, in comparison with that starting from the ordered or random state. It extends the study of the domain-wall motion at the zero or very low temperatures to the critical temperature, and especially explores the dynamic behavior far from equilibrium. In this 
paper, we intend to clarify first the dynamic scaling behavior of model A [49]. Then the dynamic theory may be generalized to model B. Along this direction, one might find the way to study relevant dynamic processes of driven diffusive lattice gases [24, 50].

Furthermore, the non-equilibrium critical dynamics around a surface is also an important topic [51 54]. For the dynamic relaxation starting from the random state, the dynamic evolution of the magnetization at surface is controlled by both the scaling dimension $x_{0}$ of the global initial magnetization and the static exponent $\beta_{1}$ of the surface magnetization [51, 52]. For the dynamic relaxation starting from the ordered state, it is expected that $\beta_{1}$ is sufficient to describe the dynamic evolution of the magnetization at surface. For the dynamic relaxation without a surface but starting from the semi-ordered state, it looks somewhat like that there exists a fictitious surface. The dynamic evolution of the magnetization inside the domain interface is governed by an exponent $\beta_{1}$. But this $\beta_{1}$ does not correspond to a static exponent in equilibrium, and it is induced by the semi-ordered initial state. Therefore, an additional purpose of this paper is to compare the dynamic relaxation starting from the semi-ordered state with that starting from the ordered state but around a surface.

In Ref. [55], brief results on the dynamic relaxation of the magnetization have been reported for the two-dimensional Ising model. This paper aims at a comprehensive study of the topic, and explores especially the dynamic scaling behavior of the Binder cumulant (or susceptibility), height function and roughness function. Furthermore, Monte Carlo simulations are performed also for the three-dimensional Ising model, to study the dimension dependence of the scaling functions and critical exponents as well as the corrections to scaling. In Sec. II, the models and scaling analysis are described, and in Sec. III, the numerical results are presented. Finally, Sec. IV includes the conclusions.

\section{MODEL AND SCALING ANALYSIS}

\section{A. Model}

The $d$-dimensional Ising model is the simplest model for magnetic materials, exhibiting a second-order phase transition. The Hamiltonian is written as

$$
-\frac{1}{k T} H=K \sum_{<i j>} S_{i} S_{j}
$$


where $S_{i}= \pm 1$ is an Ising spin at site $i$ of a square or cubic lattice, the sum is over the nearest neighbors, and $T$ is the temperature. In this paper, we set the temperature at its critical value $T_{c}$; or in other words, we set $K$ at its critical value $K_{c}$. The Hamiltonian of the Ising model itself does not include an intrinsic dynamics. For example, Monte Carlo algorithms may be introduced to simulate the dynamic evolution of the system. It is generally believed that the Monte Carlo dynamics is in the same universality class of the Langevin equation.

Let us consider a kind of dynamic relaxation processes at the critical temperature. After a macroscopic initial state at very low temperatures is prepared, the dynamic system is suddenly quenched to the critical temperature, and then released to the dynamic evolution of model A [9, 49]. For the dynamics of model A, the order parameter and other relevant physical quantities are not conserved during the dynamic evolution. In Monte Carlo simulations, it can be simply realized with a standard one-spin flip. In this paper, the heat-bath algorithm is always used in the dynamic Monte Carlo simulations. Selecting a single spin $S_{i}$, we flip it with the transition rate,

$$
P\left(S_{i} \rightarrow S_{i}^{\prime}\right) \sim \frac{\exp \left(K S_{i}^{\prime} \sum_{j(i)} S_{j}\right)}{\exp \left(K \sum_{j(i)} S_{j}\right)+\exp \left(-K \sum_{j(i)} S_{j}\right)},
$$

where $j(i)$ labels the nearest neighbors of the site $i$, and $c$ is the normalization constant. In fact, other Monte Carlo algorithms, such as Metropolis algorithms, Monte Carlo algorithms with a multi-spin flip and rejection-free Monte Carlo algorithms etc, yield the same results. The condition is that the algorithms should be local, i.e., only spins in a local region are flipped in a single flip.

With Monte Carlo simulations, we first study the critical relaxation starting from a semiordered state, taking the two-dimensional (2D) and three-dimensional (3D) Ising model as examples. The Ising model is defined on a rectangular lattice $2 L \times L$ in two dimensions and $2 L \times L^{2}$ in three dimensions, with a linear size $2 \mathrm{~L}$ in the $x$ direction and $L$ in the other directions. Periodic boundary conditions are used in all the directions. The semi-ordered state is such a state, that spins are positive on the sublattice $L^{d}(d=2$ or 3$)$ at the right side and negative on the sublattice $L^{d}$ at the left side. For convenience, we set the $x$-axis such that the domain wall between the positive and negative spins is located at $x=0$. So the $x$ coordinate of a lattice site is a half-integer.

After preparing the semi-ordered initial state, we update the spins with the heat-bath algorithm at the critical temperature $T_{c}$. Since no external magnetic field is added, macro- 
scopically the domain wall does not move. As time evolves, however, the domain wall fluctuates and creates bubbles. As a result, the domain wall becomes thicker and thicker, and a dynamic roughening process occurs. Therefore, we call it a domain interface. In Fig. 1, the dynamic evolution of the spin configuration around the domain wall is illustrated. Somewhat different from a standard growing interface, here the bulk evolves in time. In analyzing the dynamic properties of the domain interface, this must be kept in mind.

For comparison, we also perform Monte Carlo simulations of the Ising model with a free or disordered surface, but starting from the ordered state. In this case, the lattice is taken to be $L^{d}(d=2$ or 3$)$. For the free surface, a free boundary condition is used in the $x$ direction, while periodic boundary conditions are used in other directions. For convenience, we set the $x$-axis such that the free surface locates at $x=1$ or $L$. For the disordered surface, the spin $S_{i}$ on the surface couples to a random field $h_{i}$ through the interaction $-H_{i} / k T=K h_{i} S_{i}$ with the random filed $h_{i}= \pm 1$. The disordered boundary condition is only implemented in the $x$ direction, and periodic conditions are used in other directions. Since the initial state is the ordered state, the magnetization decays in time. In particular, the magnetization is also $x$-dependent due to the geometric surface. The dynamic behavior of the magnetization around the surface is governed by the surface exponents, while that at bulk is controlled by the bulk exponents. The region affected by the geometric surface grows in time, and it looks like that the surface becomes thicker and thicker. Phenomenologically, this dynamic behavior is similar to that of the domain interface.

Finally, to expose the dynamic evolution of the bulk, we perform Monte Carlo simulations of the Ising model with periodic boundary conditions in all directions, starting from the ordered state. The lattice is taken to be $L^{d}$ in $d$ dimensions.

\section{B. Scaling analysis}

We first analyze the dynamic scaling behavior of the domain interface. Due to the semiordered initial state, the time evolution of the dynamic system is inhomogeneous in the $x$ direction. Therefore, we measure the magnetization and its second moment as functions of $x$ and $t$. In two dimensions, for example,

$$
M^{(k)}(t, x)=\frac{1}{L^{k}}\left\langle\left[\sum_{y=1}^{L} S_{x y}(t)\right]^{k}\right\rangle, \quad k=1,2 .
$$


Here $S_{x y}(t)$ is the spin at the time $t$ on the lattice site $(x, y), L$ is the lattice size, and $\langle\ldots\rangle$ represents the statistical average. For convenience, we also use $M(t, x) \equiv M^{(1)}(t, x)$ to denote the magnetization. Then we can define a time-dependent Binder cumulant [9, 55],

$$
U(t, x)=\frac{M^{(2)}(t, x)}{M(t, x)^{2}}-1 .
$$

The susceptibility $M^{(2)}(t, x)-M(t, x)^{2}$ or the Binder cumulant $U(t, x)$ describes the fluctuation in the $y$ direction. In three dimensions, we simply use $S_{x y z}$ to denote the spin on the lattice site $(x, y, z)$, and similarly define the magnetization and Binder cumulant.

In order to characterize the growth of the domain interface and its fluctuation in the $x$ direction, we introduce a height function and its second moment in the $x$ direction,

$$
h^{(k)}(t)=\frac{1}{L^{k}}\left\langle\left[\sum_{x=1}^{L} S_{x y}(t)\right]^{k}\right\rangle, \quad k=1,2 .
$$

Here $\langle\ldots\rangle$ represents not only the statistic average but also the average in the $y$ direction. As usual, we also use the notation $h(t) \equiv h^{(1)}(t)$. Then the roughness function of the domain interface is defined as

$$
\omega^{2}(t)=h^{(2)}(t)-h(t) h(t) .
$$

Except for the scaling dimension of the magnetization, the height function measures the thickness of the domain interface, while the roughness function represents the fluctuation of the domain interface.

At the critical temperature $T_{c}$ and in the thermodynamic limit, there are two length scales in the dynamic system, i.e., $x$ and the non-equilibrium spatial correlation length $\xi(t)$. For a finite system, the lattice size $L$ is an additional length scale. In general, one may believe that $\xi(t)$ universally grows as $\xi(t) \sim t^{1 / z}$ in all spatial directions, because of the homogeneity of the interactions in the Hamiltonian. Therefore, general scaling arguments lead to the scaling form of the magnetization and its second moment

$$
M^{(k)}(t, x, L)=t^{-k \beta / \nu z} \widetilde{M}^{(k)}\left(t^{1 / z} / x, t^{1 / z} / L\right), \quad k=1,2 .
$$

Here $\beta$ and $\nu$ are the static exponents, and $z$ is the dynamic exponent. On the right side of the equation, the overall factors $t^{-k \beta / \nu z}$ indicates the scaling dimension of $M^{(k)}$, and the scaling function $\widetilde{M}^{(k)}\left(t^{1 / z} / x, t^{1 / z} / L\right)$ represents the scale invariance of the dynamic system. In general, the scaling form in Eq. (7) holds already in the macroscopic short-time regime, after a microscopic time scale $t_{m i c}[1,9]$. 
For the magnetization, the scaling function $\widetilde{M}\left(t^{1 / z} / x, t^{1 / z} / L\right)$ is independent of $L$ in the thermodynamic limit $L \rightarrow \infty$. Then the scaling form is simplified to

$$
M(t, x)=t^{-\beta / \nu z} \widetilde{M}\left(t^{1 / z} / x\right)
$$

For the susceptibility, it is different. For a sufficiently large lattice and in the short-time regime, the non-equilibrium spatial correlation length $\xi(t)$ is much smaller than the lattice size $L$. Therefore, the spatially correlating terms $<S_{x y_{1}} S_{x y_{2}}>-<S_{x y_{1}}><S_{x y_{2}}>$ with $\left|y_{2}-y_{1}\right|>\xi(t)$ can be neglected. In other words, one of the two summations over $y_{1}$ and $y_{2}$ in the susceptibility $M^{(2)}(t, x)-M(t, x)^{2}$ is suppressed. It then leads to the finite-size behavior $M^{(2)}(t, x)-M(t, x)^{2} \sim 1 / L^{d-1}$ (d=2 or 3) [9]. Together with Eqs. (7) and (8)), one may derive the scaling form of the Binder cumulant [9]

$$
U(t, x)=\frac{t^{(d-1) / z}}{L^{d-1}} \widetilde{U}\left(t^{1 / z} / x\right) .
$$

The Binder cumulant is interesting, for only the dynamic exponent $z$ is involved.

By definition, the height function $h(t)$ is nothing but the average magnetization in the positive domain, i.e., $h(t)=\sum_{x>0} M(t, x) / L$. In general, $h(t)$ does not obey a simple power law. Its behavior replies on the scaling function $\widetilde{M}\left(t^{1 / z} / x\right)$. In fact, one may deduce a scaling form for $h(t)$ from Eq. (17),

$$
h(t)=t^{-\beta / \nu z} \widetilde{h}\left(t^{1 / z} / L\right)
$$

Different from $M(t, x)$, here one should not ignore the dependence on the lattice size $L$, for the scaling function $\widetilde{h}\left(t^{1 / z} / L\right)$ just represents the dynamic effect of the domain interface. This is obvious from the definition $h(t)=\sum_{x>0} M(t, x) / L$. On the other hand, one should also note that the height function here is scaled as the magnetization, not a spatial length.

Similar to Eq. (10), one may also assume that the scaling form for the roughness function is $\omega^{2}(t)=t^{-2 \beta / \nu / z} F\left(t^{1 / z} / L\right)$. For later convenience, we separate a factor $t^{1 / z} / L$ from $F\left(t^{1 / z} / L\right)$, and rewrite the scaling form as

$$
\omega^{2}(t)=\frac{t^{(1-2 \beta / \nu) / z}}{L} \widetilde{\omega^{2}}\left(t^{1 / z} / L\right) .
$$

In general, $\omega^{2}(t)$ does not exhibit a power-law behavior. This is different from a standard growing interface. The reason is that here $\omega^{2}(t)$ includes fluctuations from the domain 
interface and the bulk. In fact, we will show in the next section that the scaling function $\widetilde{\omega^{2}}\left(t^{1 / z} / L\right)$ describes the fluctuation induced by the domain interface.

The scaling forms in Eqs. (7) -(11) can be also applied to the dynamic relaxation with a free or disordered surface, but starting from the ordered state. One should only keep in mind that $\beta, \nu$ and $z$ are the critical exponents at bulk. The critical exponents at surface should be deduced from the scaling functions in Eqs. (7) -(11). In this case, the lattice is taken to be $L^{d}$. The inhomogeneity in the $x$ direction is induced by the surface.

The purpose of this paper is to investigate whether the scaling forms in Eqs. (77) - (11) do hold in the dynamic relaxation with the domain interface and with the free or disordered surface. With Monte Carlo simulations, we study characteristics of the scaling functions, and extract corresponding critical exponents. Dynamic systems with the domain interface and with the free or disordered surface share some common features, although they are intrinsically different. The domain interface is induced by the geometric structure of the semi-ordered initial state, while the dynamic relaxation with the free or disordered surface is controlled by the geometric surface which remains even in equilibrium.

It is important that the height function $h(t)$ and roughness function $\omega^{2}(t)$ in Eqs. (5) and (6) include the dynamic evolution of the bulk. Therefore, their behaviors deviate from those of a standard growing interface. To obtain the dynamic features of a pure growing interface such as the power-law behavior and the roughness exponent etc, we need to subtract the contribution of the bulk. Therefore, we finally perform Monte Carlo simulations of the Ising model on a lattice $L^{d}$ with periodic boundary conditions in all directions, and starting from the ordered state. In this case, the dynamic system is homogeneous in all directions. The height function $h_{b}(t)$ and the roughness function $\omega_{b}^{2}(t)$ are just the line magnetization and its susceptibility in the $x$ or $y$ direction. The scaling functions $\widetilde{h}\left(t^{1 / z} / L\right)$ and $\widetilde{\omega^{2}}\left(t^{1 / z} / L\right)$ in Eqs. (10) and (11) are constants. In other words, $h_{b}(t)$ and $\omega_{b}^{2}(t)$ show a power-law behavior.

Then we may redefine the pure height function and roughness function for the dynamic relaxation of the domain interface or around the surface by subtracting the contribution from the bulk

$$
\begin{gathered}
D h(t, L)=h_{b}(t)-h(t), \\
D \omega^{2}(t, L)=\omega^{2}(t)-\omega_{b}^{2}(t) .
\end{gathered}
$$

We may expect that $D h(t, L)$ and $D \omega^{2}(t, L)$ exhibit a power-law behavior as in the case of 
a standard growing interface. Here we should note that we define $D h(t, L)$ as $h_{b}(t)-h(t)$ rather than $h(t)-h_{b}(t)$, for $h(t)$ decays in time faster than $h_{b}(t)$.

\section{MONTE CARLO SIMULATION}

For the 2D Ising model, our main results are presented with $L=512$ at $K_{c}=0.44069$, and the maximum updating time is $t_{M}=25600$. Additional simulations with $L=1024$ and $L=256$ are performed, to investigate the finite-size scaling behavior and finite-size effect. The total samples for average are 24000. For the 3D Ising model, the main results are obtained with $L=128$ at $K_{c}=0.22165$, and the maximum updating time is $t_{M}=2560$. Additional simulations with $L=256$ and $L=64$ are performed to investigate the finitesize scaling behavior and finite-size effect. The total samples for average are 30000. The statistical errors are estimated by dividing the total samples into two or three subgroups. If the fluctuation in the time direction is comparable with or larger than the statistical error, it will be taken into account.

Theoretically, the scaling forms described in the previous section hold in the macroscopic short-time regime, after a microscopic time scale $t_{m i c} . t_{m i c}$ is not universal, and relies on microscopic details of the dynamic systems. In Monte Carlo simulations, for example, $t_{m i c}$ is typically tens or hundreds of Monte Carlo time steps [9]. With quenched disorder or frustration, $t_{m i c}$ could be longer. For the simple Ising model with the nearest neighbor interactions, $t_{m i c}$ is rather short, about 10 - 20 time steps. Therefore, critical exponents are typically obtained in the time intervals [20,25600] in two dimensions and [10,2560] in three dimensions. From the data collapse of different $x$ and $L$, one may observe the scaling functions in a even larger time window.

\section{A. Magnetization}

The time evolution of the magnetization of the 2D Ising model starting from the semiordered state is displayed in Fig. 2. For a sufficiently small $s$, e.g., $x=255.5$ and $t<t_{M}=$ 25600, $M(t, x)$ approaches the non-linear decay at bulk, $M(t, x) \sim t^{-\beta / \nu z}[9]$. The exponent $\beta / \nu z=0.0580(3)$ measured from the slope of the curve is well consistent with $\beta=1 / 8$, $\nu=1$ and $z=2.16(1)$ reported in the literature [9]. For a sufficiently large $s$, e.g., $x=0.5$ 
and $t>20, M(t, x)$ exhibits also a power-law behavior, but decays much faster than at bulk. In other words, we catch some features of the scaling function $\widetilde{M}(s)$ in Eq. (8),

$$
\widetilde{M}(s) \sim\left\{\begin{array}{ll}
\text { const } & s \rightarrow 0 \\
s^{-\beta_{0} / \nu} & s \rightarrow \infty
\end{array},\right.
$$

with $s=t^{1 / z} / x$. In the limit $s \rightarrow \infty$, one may define an interface exponent $\beta_{1}$ such that

$$
M(t, x) \sim t^{-\beta_{1} / \nu z} \cdot x^{\beta_{0} / \nu}, \quad \beta_{1}=\beta+\beta_{0} .
$$

Inside the interface, the power-law decay of the magnetization is governed by the interface exponent $\beta_{1}$, while outside the interface, it is controlled by the bulk exponent $\beta$. In Fig. 2, one measures $\beta_{1} / \nu z=0.518(4)$, and then calculates $\beta_{1}=1.119(9)$ and $\beta_{0} / \nu=0.994(9)$. Similar to the exponent $x_{0}$ in the dynamic relaxation starting from the random state [1, 9], $\beta_{0}$ here is induced by the semi-ordered initial state. Accounting the error, one may believe $\beta_{0} / \nu=1$, which suggests that $M(t, x)$ is an analytic function of $x$. This result is also supported by the simulations of the $3 \mathrm{D}$ Ising model. Since $\beta_{1}$ is much bigger than $\beta$, the magnetization inside the domain interface decays much faster than that at bulk. This phenomenon is understandable, for the dynamic evolution of the spins in the positive domain is strongly affected by those in the negative domain, and vice versa.

To fully confirm the scaling form in Eq. (8) , for example, we fix $x^{\prime}=1.5$, and change the time scale $t$ of another $x$ to $\left(x^{\prime} / x\right)^{z} t$, and the scale of $M(t, x)$ to $\left(x^{\prime} / x\right)^{-\beta / \nu} M(t, x)$. As shown in Fig. 2, all data of different $x$ nicely collapse to the curve of $x^{\prime}=1.5$. This validates Eq. (8)). Alternatively, we may plot $t^{\beta / \nu z} M(t, x)$ as a function of $s=t^{1 / z} / x$. According to Eq. (8), all data of different $x$ should collapse onto the master curve $\widetilde{M}(s)$. This is shown in Fig. 3. Clearly, $\widetilde{M}(s) \rightarrow$ const when $s \rightarrow 0$, while $\widetilde{M}(s) \rightarrow s^{-\beta_{0} / \nu}$ when $s \rightarrow \infty$.

For comparison, the time evolution of the magnetization of the 2D Ising model with a free surface but starting from the ordered state is shown in Fig. 4, For a sufficiently small $s$, e.g., $x=256$ and $t<t_{M}=25600, M(t, x)$ approaches also the non-linear decay at bulk, $M(t, x) \sim t^{-\beta / \nu z}$ with $\beta / \nu z=0.0579(4)$. For a sufficiently large $s$, e.g., $x=1$ and $t>20, M(t, x)$ exhibits also a power-law behavior. Assuming again the scaling ansatzes in Eqs. (14) and (15), the measurement of the slope yields $\beta_{1} / \nu z=0.231(1)$. Then one calculates $\beta_{1}=0.499(2)$. It is in good agreement with the surface exponent $\beta_{s}=1 / 2$ for the free surface [56]. Now, the exponent $\beta_{0} / \nu$ is estimated to be 0.374 . Therefore, $M(t, x)$ 
is not an analytic function of $x$, when $x$ approaches the free surface. This is very different from the domain interface. Finally, the data collapse according to Eq. (8) is also shown in Fig. 专, and it fully confirms the scaling form.

As in Figs. 22 and 4, similar analysis can be carried out for the magnetization of the 2D Ising model with a disordered surface. For a small $s$, one measures $\beta / \nu z=0.0578(7)$. For a large $s$, careful analysis shows that the power-law behavior is not perfect [55]. In the equilibrium state, one may show that the surface exponent $\beta_{s}$ of the disordered surface remains $1 / 2$, but with a logarithmic correction to scaling [56]. Therefore, we fit the timedependent magnetization at $x=0.5$ with a logarithmic correction to scaling, i.e., $M(t)=$ $c_{1} t^{-\alpha_{1}} /\left(1+c_{2} \ln (t)\right)^{1 / 2}$, and derive $\alpha_{1}=0.231$, consistent with $\beta_{1} / \nu z=0.231(1)$ for the free surface. If one fixes $c_{2}=0$, it yields $\alpha_{1}=0.272$, significantly different from 0.231(1).

In Fig. 3, the scaling function $\widetilde{M}(s)$ with $s=t^{1 / z} / x$ is plotted for the domain interface, free surface and disordered surface. We clearly observe the characteristic of the scaling function in Eq. (14), and measure the exponent $\beta_{0}=0.998(5)$ for the domain interface, and $0.372(6)$ for the free surface. Due to the logarithmic correction to scaling, $\widetilde{M}(s)$ of the disordered surface decays faster than that of the free surface at the large $s$ regime.

We emphasize that in the case of the free surface or disordered surface, the exponent $\beta_{1} \equiv \beta_{s}$ does describe the critical behavior of the magnetization at the surface in equilibrium. Around the free surface, for example, $M(\tau) \sim(-\tau)^{\beta_{1}}$ with $\tau$ being the reduced temperature. It is important that $\beta_{1}$ is induced by the geometric surface which remains forever. In the case of the domain interface, however, $\beta_{1}$ is induced by the geometric structure of the semiordered initial state. When the dynamic system reaches its equilibrium state, the influence of the initial state disappears and the critical behavior of the magnetization is governed by the bulk exponent $\beta$ everywhere. We should keep in mind, however, that exactly at the critical temperature (i.e., $\tau=0$ ) and in the thermodynamic limit, the dynamic system never reaches its equilibrium state in a finite time due to the divergent correlating time. According to Eq. (8), therefore, the domain interface and the free or disordered surface behave similarly.

For the 3D Ising model, the static and dynamic exponents at bulk are known to be $\beta=0.327(1), \nu=0.630(2)$ and $z=2.04(1)$ [57]. For the free surface, the surface exponent is $\beta_{s}=0.795(10)$ [58]. Following the procedure for the 2D Ising model, we have analyzed the scaling behavior of the dynamic relaxation with the domain interface, free surface and disordered surface. Especial attention is drawn to the critical exponent $\beta_{0} / \nu$. 
Let us first consider the domain interface. For a small $s$, the magnetization shows the power-law behavior at bulk, $M(t, x) \sim t^{-\beta / \nu z}$. The critical exponent is estimated to be $\beta / \nu z=0.253(5)$, well consistent with the value 0.253(1) at bulk [57]. For a large $s$, e.g., $x=0.5$ and $t>10$, the magnetization exhibits the power-law behavior $M(t, x) \sim t^{-\beta_{1} / \nu z}$ with $\beta_{1}=\beta+\beta_{0}$ in Eq. (15). From the slope of the curve, one obtains $\beta_{1} / \nu z=0.744(2)$. Then one calculates the critical exponent $\beta_{0} / \nu=(0.744-0.253) \times 2.04=1.002(4)$. For the 2D Ising model, $\beta_{0} / \nu=0.998(5)$. These two measurements of $\beta_{0} / \nu$ strongly suggest $\beta_{0} / \nu=1$, and it is dimension-independent. Therefore $M(t, x)$ is an analytic function of $x$. In Fig. 5, the scaling functions $\widetilde{M}(s)$ of the magnetization for the 3D Ising model is plotted. Data collapse for different $x$ is observed. From the slope of the curve in the large $s$ regime, one measures $\beta_{0} / \nu=1.001(6)$.

Similar analysis is applied to the magnetization of the 3D Ising model with the free and disordered surfaces, and the scaling function is also shown in Fig. 5, Different from the case of the 2D Ising model, the large- $s$ tails of the scaling function $\widetilde{M}(s)$ for the free and disordered surfaces look parallel each other. In the inset of the figure, the magnetization at $x=1.0$ is displayed for both the free and disordered surfaces. The slope is $\beta_{1} / \nu z=0.623(5)$ for the free surface, and $0.632(2)$ for the disordered surface. The difference is only one or two per cent, and the correction to scaling is rather small. For the free surface, one estimates $\beta_{1}=0.623(5) \times \nu z=0.801(6)$. Alternatively, one may also measure $\beta_{0} / \nu=0.747(6)$ from the scaling function obtained with different $x$, and then calculates $\beta_{1}=\beta+\beta_{0}=0.798(4)$. These values of $\beta_{1}$ are well consistent with the surface exponent $\beta_{s}=0.795(10)$ [58].

\section{B. Binder Cumulant}

In Fig. 6, the time evolution of the Binder cumulant is displayed for the 2D Ising model starting from the semi-ordered state. For a sufficiently small $s$, e.g., $x=255.5$ and $t<t_{M}=$ 25600, the Binder cumulant exhibits the power-law behavior at bulk, $U(t, x) \sim t^{(d-1) / z}$. From the slope, one measures $(d-1) / z=0.468(4)$, and it is consistent with $(d-1) / z=0.463(3)$ calculated from $z=2.16(1)$. For a sufficiently large $s$, e.g., $x=0.5$ and $t>20$, the Binder cumulant grows also by a power law, but much faster than that at the large $x$. Then we 
extract the characteristic of the scaling function $\widetilde{U}(s)$,

$$
\widetilde{U}(s) \sim\left\{\begin{array}{ll}
\text { const } & s \rightarrow 0 \\
s^{d_{0}} & s \rightarrow \infty
\end{array} .\right.
$$

In the limit $s \rightarrow \infty$, one may derive from Eqs. (9) and (16),

$$
U(t, x) \sim t^{\left(d-1+d_{0}\right) / z} /\left(L^{d-1} x^{d_{0}}\right)
$$

From the curve of $x=0.5$ in Fig. 6, one estimates $\left(d-1+d_{0}\right) / z=1.390(8)$. Taking $z=2.16$ as input, one calculates $d_{0}=1.390 \times 2.16-(d-1)=2.00(2)$, very close to 2 .

In Fig. 7, the Binder cumulant is plotted for the 3D Ising model starting from the semiordered state. For a small $s$, one observes the power-law behavior at bulk, $U(t, x) \sim t^{(d-1) / z}$. From the slope of the curve, one obtains 0.995(12), and then estimates $z=2.01(2)$, consistent with $z=2.04(1)$ from the literature [57]. For a large $s$, e.g., $x=0.5$ and $t>10$, one estimates $\left(d-1+d_{0}\right) / z=1.963(10)$ from the power-law behavior in Eq. (17), then derives $d_{0}=2.01(2)$. Again it is close to 2. To further verify the scaling form in Eq. (9), we fix $x^{\prime}=1.5$, and change the time scale $t$ of another $x$ to $\left(x^{\prime} / x\right)^{z} t$, and the scale of $U(t, x)$ to $\left(x^{\prime} / x\right)^{d-1} U(t, x)$. As shown in Fig. 6 and 7, all curves of different $x$ nicely collapse to the curve of $x^{\prime}=1.5$. This fully confirms the scaling form in Eq. (9)).

To reveal the lattice-size dependence of the Binder cumulant in Eq. (9), we fix $x=0.5$, and plot $U(t, L) \equiv U(t, x=0.5)$ as a function of $t$ for different $L$ in Fig. 8. Obviously, all curves of different $L$ and in two- and three-dimensions are parallel each other, and exhibit the power-law behavior in Eq. (17). We then fix a lattice size $L^{\prime}$, and change the scale of $U(t, L)$ of another $L$ to $U(t, L)\left(L^{\prime} / L\right)^{-(d-1)}$. Data collapse is clearly observed for both the $2 \mathrm{D}$ and $3 \mathrm{D}$ Ising models.

In Fig. 9, the scaling function $\widetilde{U}(s)$ with $s=t^{1 / z} / x$ is plotted for the 2D Ising model with the domain interface and free surface. Data of different $x$ collapse clearly onto their master curves. For the domain interface, the asymptotic behavior of $\widetilde{U}(s)$ in Eq. (16) is exposed. The exponent $d_{0}$ is measured to be 2.00(2), the same as that extracted from the single curve of $x=0.5$ in Fig. [6. For the free surface, $\widetilde{U}(s) \rightarrow$ const is also observed in the limit $s \rightarrow 0$. In the large $s$ regime, however, it does not exhibit a power-law behavior. Instead, it increases by a logarithmic law, $\widetilde{U}(s)=a_{0}+a_{1} \ln (s)$. In other words, the exponent $d_{0}$ of the free surface is effectively 0 but with a logarithmic correction. This result indicates 
that the spatial fluctuation of the domain interface grows in time much faster than that of the free surface.

In three dimensions, $d-1-2 \beta_{1} / \nu$ of the free surface is negative. Starting from an ordered state, the susceptibility decays in time. Therefore, one suffers from large fluctuations, and it is difficult to address the dynamic behavior of the susceptibility or Binder cumulant. For the disordered surface, the situation is even more complicated. Since our paper is already lengthy, we will not go into the details here.

\section{Height function and roughness function}

In the preceding two subsections, we have analyzed the temporal and spatial structures of the magnetization $M(t, x)$ and Binder cumulant $U(t, x)$. Up to now, however, we have not yet touched how the interface grows and fluctuates in the $x$ direction. For this purpose, we have introduced the height function $h(t)$ and the roughness function $\omega^{2}(t)$ in Eqs. (5) and (6) . For a standard growing interface, the time evolution of the height function $h(t)$ may be not so important, but the roughness function $\omega^{2}(t)$ exhibits a power-law behavior governed by the roughness exponent.

In Fig. 10, the height function $h(t)$ is plotted for the 2D Ising model. One finds a powerlaw behavior for the dynamic relaxation of the bulk, i.e., with periodic boundary conditions in all directions. The slope of the curve is $0.0576(3)$, consistent with $\beta / \nu z=0.0579(3)$ from the literature [9]. For the domain interface and free surface, the height function decreases faster than a power law. Actually, the curves can be fitted by a double power law, e.g., $h(t)=c_{0} t^{\alpha_{0}}-c_{1} t^{\alpha_{1}}$. Although this four-parameter fit could not produce very accurate values of $\alpha_{0}$ and $\alpha_{1}$, it leads us to introduce the pure height function $D h(t, L)$ in Eq. (12). The

conjecture is that the term $c_{1} t^{\alpha_{1}}$ represents the pure interface, and $c_{0} t^{\alpha_{0}}$ is the magnetization of the bulk. In Fig. 10, we do observe a power-law behavior for the pure height function $D h(t, L)$. The slope of the curves is estimated to be $0.407(2)$. In comparison with that for a standard growing interface, this power-law behavior is special for the domain interface.

In Fig. 11, the roughness function is plotted for the 2D Ising model. In the case of the bulk, $\omega^{2}(t)$ is expected to obey a power law, although there exist corrections to scaling. Anyway, one may roughly estimate the exponent $(1-2 \beta / \nu) / z$ to be $0.345(14)$, consistent with $z=2.16(1)$. Due to corrections to scaling, the dynamic behavior of $\omega^{2}(t)$ looks unclear 
for the domain interface and free surface. However, the pure roughness function $D \omega^{2}(t, L)$ in Eq. (13) obviously obeys a power-law behavior for both the domain interface and free surface. It seems that $\omega^{2}(t)$ and $\omega_{b}^{2}(t)$ have the same correction to scaling. Neglecting the corrections to scaling, one may assume

$$
\widetilde{\omega^{2}}(u)=\left\{\begin{array}{ll}
\mathrm{c} & \text { without interface } \\
c+u^{d_{\omega}} & \text { with interface }
\end{array} .\right.
$$

Then one derives

$$
D \omega^{2}(t, L)=\frac{t^{\left(1-2 \beta / \nu+d_{\omega}\right) / z}}{L^{1+d_{\omega}}} .
$$

In Fig. 11, the curves of $D \omega^{2}(t, L)$ for the domain interface and free surface are parallel each other. From the slopes of the curves one measures $\left(1-2 \beta / \nu+d_{\omega}\right) / z=0.808(2)$. Then one calculates the exponent $d_{\omega}=0.995(4)$. The fluctuations of the domain interface and free surface grow faster in time than that of the bulk. More importantly, the exponent $\left(1-2 \beta / \nu+d_{\omega}\right) / z$ is independent of the interface exponent or surface exponent $\beta_{1}$.

One may also verify the lattice-size dependence, $D \omega^{2}(t, L) \sim 1 / L^{1+d_{\omega}}$ in Eq. (19). In Fig. 12, $D \omega^{2}(t, L)$ is plotted for different lattice sizes. Obviously, all the curves are parallel each other. Then we fix a lattice size, e.g., $L^{\prime}=256$, and change the scale of $D \omega^{2}(t, L)$ of another $L$ to $D \omega^{2}(t, L)\left(L^{\prime} / L\right)^{-\left(1+d_{\omega}\right)}$. Taking $d_{\omega}=1$ as input, data collapse is clearly observed.

For a standard growing interface, the roughness function grows by $\omega^{2}(t) \sim t^{2 \alpha / z}$, and $\alpha$ is the so-called roughness exponent. According to Eq. (19), the roughness exponent for the domain interface and free surface is $\alpha=\left(1-2 \beta / \nu+d_{\omega}\right) / 2=0.872(8)$. On the other hand, from the dimension counting one may expect $D h(t, L) \sim t^{\alpha / z}$ for the pure height function. From the measurement $\alpha / z=0.407(2)$ in Fig. 10, one calculates $\alpha=0.879(6)$. These two measurements of the exponent $\alpha$ are in good agreement with each other. In the scaling analysis of $D \omega^{2}(t, L),-\beta / \nu$ represents the scaling dimension of the magnetization. One may remove it, e.g., by dividing $D \omega^{2}(t, L)$ by $M(t)^{2}$ of the bulk. Then the real roughness exponent is $\left(1+d_{\omega}\right) / 2$. Since $d_{\omega}=1$, the roughness exponent is just 1 . This conclusion holds also for the Ising model in three dimensions. But the measurements of the exponents are somewhat complicated in this case, for $1-2 \beta / \nu=-0.038$ is around zero. 


\section{CONCLUSION}

In summary, we have investigated the non-equilibrium critical dynamics with a domain interface, a free surface and a disordered surface, taking the two- and three-dimensional Ising models as examples. The dynamic scaling behavior is revealed, and a dynamic roughening process is observed. Critical exponents characterizing the magnetization, Binder cumulant, height function and roughness function are extracted, and the results are summarized in Table I.

i) For the domain interface, $\beta_{0} / \nu$ for the magnetization in Eq. (14) takes values close to 1 for both the two- and three-dimensional Ising models. It indicates that the magnetization $M(t, x)$ is an analytic function of $x$. Especially, $M(t, x)$ inside the domain interface decays much faster in time than that at bulk, for the interface exponent $\beta_{1}=\beta+\beta_{0}$ is much bigger than the bulk exponent $\beta$. For the free surface, the values of $\beta_{1} \equiv \beta_{s}$ are in agreement with the measurements in equilibrium. For the disordered surface, $\beta_{1} \equiv \beta_{s}^{*}$ takes the same value as that of the free surface, but with a logarithmic correction to scaling in two dimensions.

ii) For the domain interface, the exponent $d_{0}$ for the Binder cumulant in Eq. (16) takes values close to 2 in two and three dimensions. For the free surface, $d_{0} \equiv d_{s}$ is effectively 0 in two dimensions, but with a logarithmic correction to scaling. These results indicate that the fluctuation in the $y$ direction inside the domain interface is stronger than that around the free surface. In fact, one can derive from Eqs. (14) and (16) that inside the domain interface, the susceptibility behaves as $M^{(2)}(t, x)-M(t, x)^{2} \sim t^{(d-1-2 \beta / \nu) / z}$, the same as that

at bulk. Around the free surface, the susceptibility is $M(t, x)^{(2)}-M(t, x)^{2} \sim t^{\left(d-1-2 \beta_{1} / \nu\right) / z}$, different from that at bulk.

iii) For both the domain interface and free surface, the roughness function in Eq. (11) does not obey a power law, for it includes the fluctuation of the bulk and domain interface. After subtracting the contribution of the bulk, the pure roughness function in Eq. (13) does exhibit a power-law behavior in Eq. (19), and the roughness exponent is identified to be $\alpha=\left(1+d_{\omega}\right) / 2$. Interestingly, the exponent $d_{\omega}$ takes values close to 1 for both the domain interface and free surface, and also independently of the spatial dimension. In other words, the fluctuation of the interface in the $x$ direction is independent of the exponent $\beta_{1}$.

Theoretically, above results need further understanding. For example, it is a challenge to derive the dynamic scaling forms with renormalization group methods. It is also important 
to investigate how the quenched disorder may affect the domain-wall motion at the critical temperature. The techniques used in this paper may be also applied to similar dynamic systems.

Acknowledgements: This work was supported in part by NNSF (China) under grant No. 10325520.

[1] H.K. Janssen, B. Schaub, and B. Schmittmann, Z. Phys. B 73, 539 (1989).

[2] D. Huse, Phys. Rev. B 40, 304 (1989).

[3] K. Humayun and A. J. Bray, J. Phys. A24, 1915 (1991).

[4] D. Stauffer, Physica A 186, 197 (1992).

[5] N. Ito, Physica A196, 591 (1993).

[6] Z.B. Li, U. Ritschel, and B. Zheng, J. Phys. A27, L837 (1994).

[7] Z.B. Li, L. Schülke, and B. Zheng, Phys. Rev. Lett. 74, 3396 (1995).

[8] H.J. Luo, L. Schülke, and B. Zheng, Phys. Rev. Lett. 81, 180 (1998).

[9] B. Zheng, Int. J. Mod. Phys. B12, 1419 (1998), review article.

[10] B. Zheng, M. Schulz, and S. Trimper, Phys. Rev. Lett. 82, 1891 (1999).

[11] R. E. Blundell, K. Humayun, and A. J. Bray, J. Phys. A25, L733 (1992).

[12] A.A. Fedorenko and S. Trimper, Europhys. Lett. 74, 89 (2006).

[13] H. Rieger, J. Phys. A26, L615 (1993).

[14] H.J. Luo, L. Schülke, and B. Zheng, Mod. Phys. Lett. B13, 417 (1999).

[15] B. Zheng, F. Ren, and H. Ren, Phys. Rev. E68, 046120 (2003).

[16] Y. Ozeki and N. Ito, Phys. Rev. B68, 054414 (2003).

[17] E. Granato and D. Dominguez, Phys. Rev. B71, 094521 (2005).

[18] Q.M. Nie, M.B. Luo, and Q.H. Chen, Phys. Rev. B74, 024523 (2006).

[19] J.Q. Yin, B. Zheng, and S. Trimper, Phys. Rev. E70, 056134 (2004).

[20] J.Q. Yin, B. Zheng, and S. Trimper, Phys. Rev. E72, 036122 (2005).

[21] Y. Ozeki and K. Ogawa, Phys. Rev. B71, 220407 (2005).

[22] Y. Chen and Z.B. Li, Phys. Rev. B71, 174433 (2005).

[23] J.Q. Yin, B. Zheng, V.V. Prudnikov, and S. Trimper, Euro. Phys. J. B49, 195 (2006).

[24] E.V. Albano and G. Saracco, Phys. Rev. Lett. 88, 145701 (2002). 
[25] B.C.S. Grandi and W. Figueiredo, Phys. Rev. E70, 056109 (2004).

[26] K. Laneri, A.F. Rozenfeld, and E.V. Albano, Phys. Rev. E72, 065105 (2005).

[27] E. Arashiro, J.R.D de Felicio, and U.H.E. Hansmanno, Phys. Rev. E73, 040902 (2006).

[28] H.K. Lee and Y. Okabe, Phys. Rev. E71, 015102 (2005).

[29] L. Schülke and B. Zheng, Phys. Rev. E62, 7482 (2000).

[30] R.A. Monetti and E.V. Albano, Europhys. Lett. 56, 400 (2001).

[31] G.P. Saracco and E.V. Albano, J. Chem. Phys. 118, 4157 (2003).

[32] C. Godréche and J.M. Luck, J. Phys.:Condens. Matter 14, 1589 (2002).

[33] M. Henkel, M. Paessens, and M. Pleimling, Phys. Rev. E69, 056109 (2004).

[34] A. Picone and M. Henkel, Nucl. Phys. B688, 217 (2004).

[35] G. Schehr and R. Paul, Phys. Rev. E72, 016105 (2005).

[36] M. Pleimling and A. Gambassi, Phys. Rev. B71, 180401(R) (2005).

[37] P. Calabrese and A. Gambassi, J. Phys. A38, R133 (2005).

[38] X.W. Lei and B. Zheng, Phys. Rev. E75, 040104 (2007).

[39] I.F. Lyuksyutov, T. Nattermann, and V. Pokrovsky, Phys. Rev. B59, 4260 (1999).

[40] T. Nattermann, V. Pokrovsky, and V.M. Vinokur, Phys. Rev. Lett. 87, 197005 (2001).

[41] A. Glatz, T. Nattermann, and V. Pokrovsky, Phys. Rev. Lett. 90, 047201 (2003).

[42] S. Miga, J. Dec, W. Kleemann, and R. Pankrath, Phys. Rev. B70, 134108 (2004).

[43] T. Braun, W. Kleemann, J. Dec, and P.A. Thomas, Phys. Rev. Lett. 94, 117601 (2005).

[44] W. Kleemann, J. Dec, V.V. Shvartsman, Z. Kutnjak, and T. Braun, Phys. Rev. Lett. 97, $065702(2006)$.

[45] W. Kleemann, J. Rhensius, O. Petracic, J. Ferré, J.P. Jamet, and H. Bernas, Phys. Rev. Lett. 99, 097203 (2007).

[46] U. Nowak and K. D. Usadel, Europhys. Lett. 44, 634 (1998).

[47] L. Roters, S. Lübeck, and K. D. Usadel, Phys. Rev. E63, 026113 (2001).

[48] M. Jost and K. D. Usadel, Phys. Rev. B54, 9314 (1996).

[49] P.C. Hohenberg and B.I. Halperin, Rev. Mod. Phys. 49, 435 (1977).

[50] S. Caracciolo, A. Gambassi, M. Gubinelli, and A. Pelissetto, Phys. Rev. Lett. 92, 029601 (2004).

[51] U. Ritschel and P. Czerner, Phys. Rev. Lett. 75, 3882 (1995).

[52] M. Pleimling, J. Phys. A37, R79 (2004). 
[53] M. Pleimling and F. Iglói, Phys. Rev. Lett. 92, 145701 (2004).

[54] M. Pleimling and F. Iglói, Phys. Rev. B71, 094424 (2005).

[55] N.J. Zhou and B. Zheng, Europhys. Lett. 78, 56001 (2007).

[56] M. Pleimling, F.A. Bagaméry, L. Turban, and F. Iglói, J. Phys. A37, 8801 (2004).

[57] A. Jaster, J. Mainville, L. Schülke, and B. Zheng, J. Phys. A32, 1395 (1999).

[58] S. V. Belim, J. Exp. and Theor. Phys. 103, 611 (2006).

\begin{tabular}{|c|c|c|c|}
\hline & & 2D Ising & 3D Ising \\
\hline \multirow[t]{4}{*}{$M(t)$} & $\beta_{0} / \nu$ & $0.998(5)$ & $1.001(6)$ \\
\hline & $\beta_{1}$ & $1.123(5)$ & $0.958(6)$ \\
\hline & $\beta_{1} \equiv \beta_{s}$ & $0.499(4)$ & $0.801(6)$ \\
\hline & $\beta_{1} \equiv \beta_{s}^{*}$ & 0.499 & $0.812(4)$ \\
\hline \multirow[t]{2}{*}{$U(t)$} & $d_{0}$ & $2.00(2)$ & $2.01(2)$ \\
\hline & $d_{s}$ & 0 & \\
\hline \multirow[t]{5}{*}{$\omega^{2}(t)$} & $d_{\omega}$ & $0.995(4)$ & 1 \\
\hline & $\beta_{s}$ & $1 / 2$ & $0.795(10)[58]$ \\
\hline & $\beta$ & $1 / 8$ & $0.327(1) \quad[57]$ \\
\hline & $\nu$ & 1 & $0.630(2)$ \\
\hline & $z$ & $2.16(1)$ [9] & $2.04(1)$ \\
\hline
\end{tabular}

TABLE I: In the upper sector, the exponents $\beta_{0} / \nu, \beta_{1}$ and $d_{0}$ are for the domain interface, $\beta_{s}$ and $d_{s}$ are for the free surface, and $\beta_{s}^{*}$ is for the disordered surface. The exponent $d_{\omega}$ is for both the domain interface and free surface. In the measurements of $\beta_{s}^{*}$ and $d_{s}$ for the 2D Ising model, logarithmic corrections to scaling are taken into account. In the lower sector, the static exponents $\beta$ and $\nu$, the dynamic exponent $z$, and the surface exponent $\beta_{s}$ are taken from the literatures. 


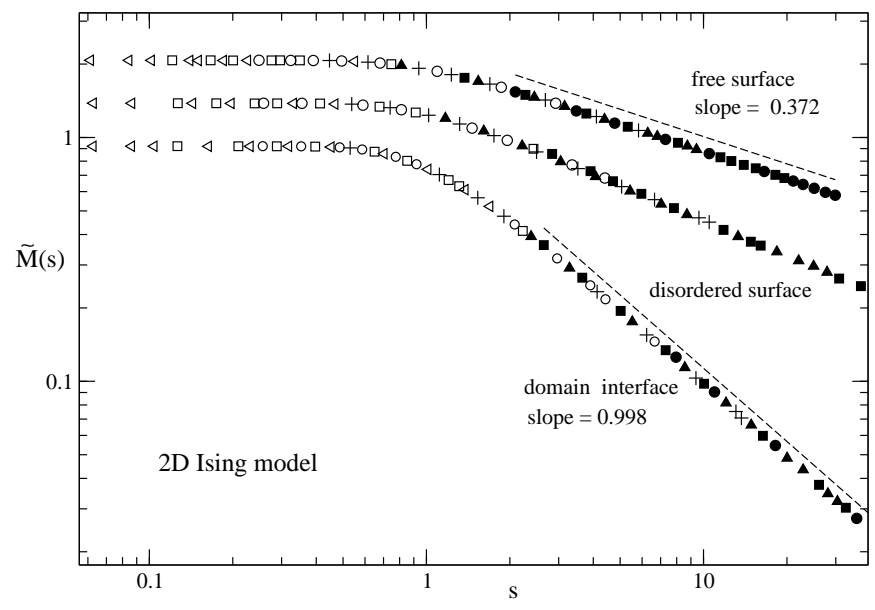

FIG. 3: The scaling functions $\widetilde{M}(s)$ with $s=t^{1 / z} / x$ is plotted on a double-log scale, for the 2D Ising model with the free surface, disordered surface and domain interface (from above). Data collapse for different $x$ is observed. Dashed lines show the power-law fits. From Ref. [55], this figure is reproduced by permission of Europhys. Lett..

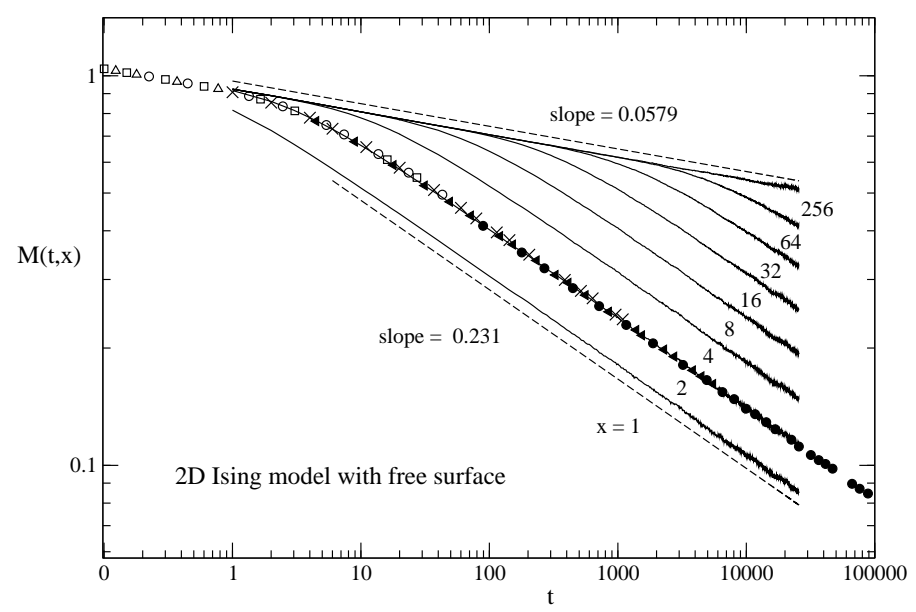

FIG. 4: The magnetization of the 2D Ising with a free surface and starting from an ordered state, is plotted with solid lines on a double-log scale. Dashed lines show the power-law fits. According to Eq. (8), data collapse for different $x$ is demonstrated at a fixed $x^{\prime}=2$. Solid circles, solid triangles, pluses, open circles, open squares and open triangles correspond to $x=1,4,8,16,32$ and 64 respectively. 


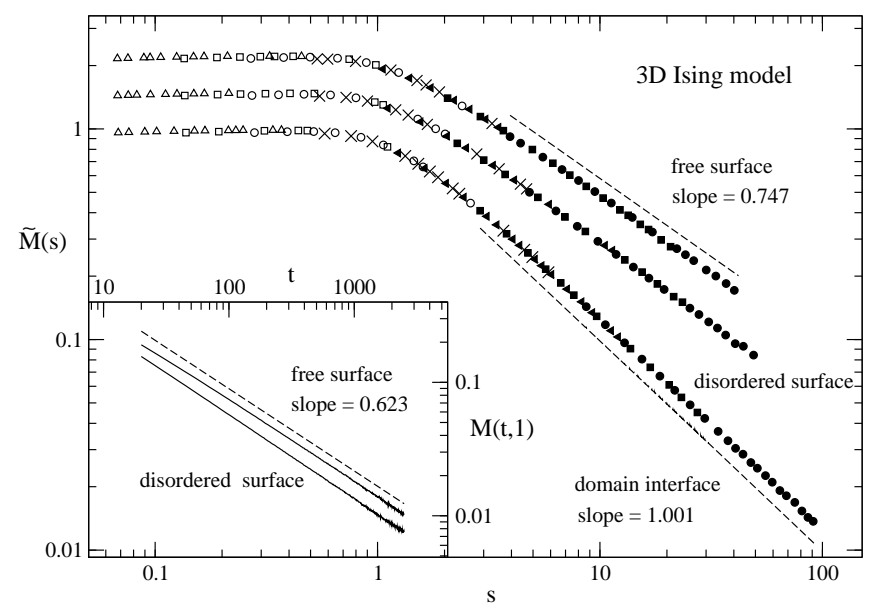

FIG. 5: The scaling function $\widetilde{M}(s)$ with $s=t^{1 / z} / x$ is plotted on a double-log scale, for the 3D Ising model with the free surface, disordered surface and domain interface (from above). Data collapse for different $x$ is observed. Dashed lines show the power-law fits. No logarithmic correction is detected for the disordered surface. In the inset, the magnetization at $x=1.0$ for the disordered surface and free surface are shown.

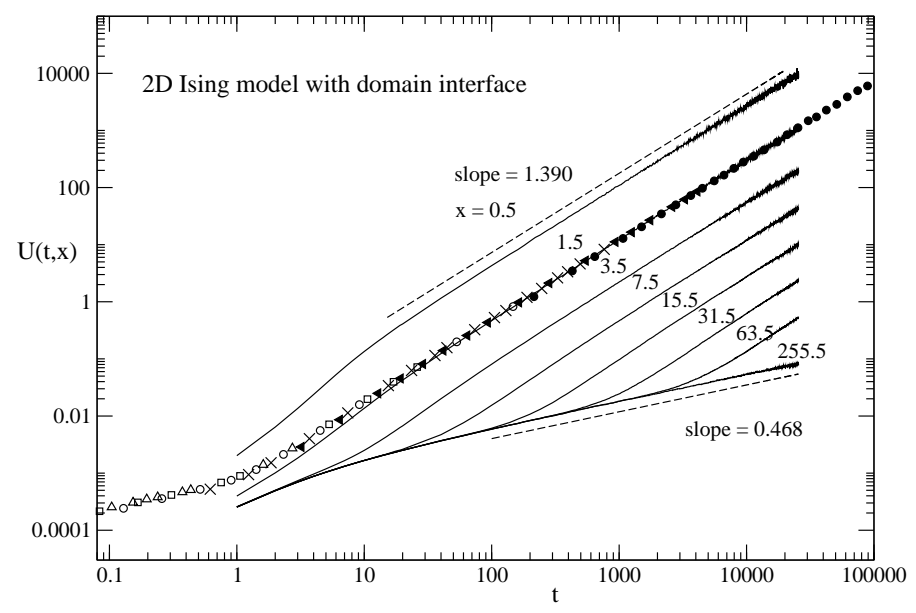

FIG. 6: The Binder cumulant of the 2D Ising model with the domain interface is plotted with solid lines on a double-log scale. Dashed lines show the power-law fits. According to Eq. (9), data collapse for different $x$ is demonstrated at a fixed $x^{\prime}=1.5$. Solid squares, solid triangles, pluses, open circles, open squares and open triangles correspond to $x=0.5,3.5,7.5,15.5,31.5$ and 63.5 respectively. 


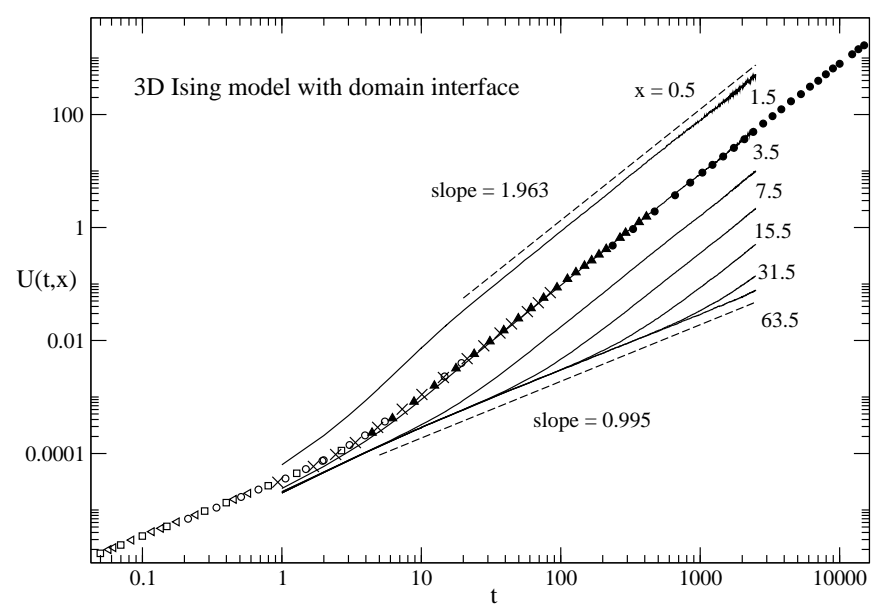

FIG. 7: The Binder cumulant of the 3D Ising model with the domain interface is plotted with solid lines on a double-log scale. Dashed lines show the power-law fits. According to Eq. (9), data collapse for different $x$ is demonstrated at a fixed $x^{\prime}=1.5$. Solid circles, solid triangles, pluses, open circles, open squares and open triangles correspond to $x=0.5,3.5,7.5,15.5,31.5,63.5$ respectively.

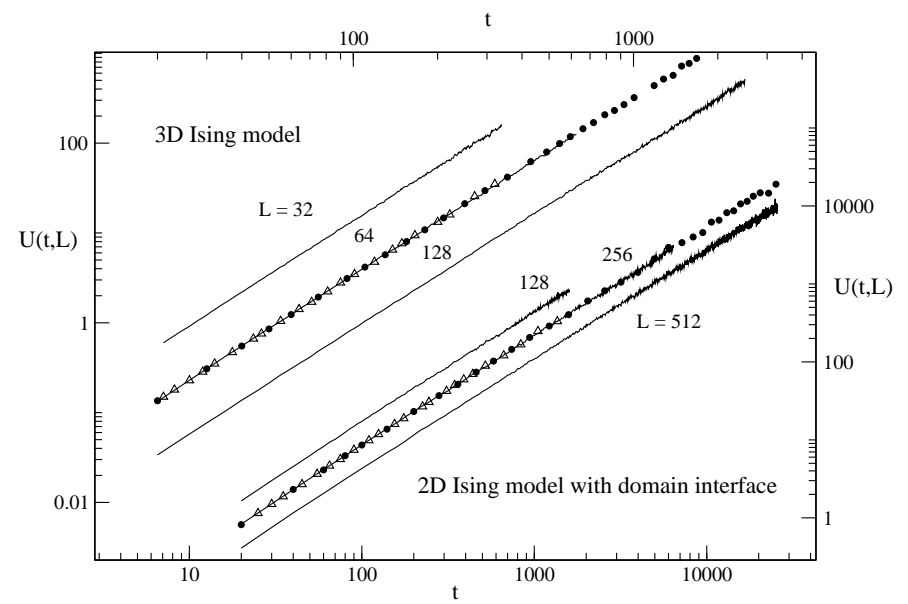

FIG. 8: The Binder cumulant at $x=0.5$ for the Ising model with the domain interface is plotted with solid lines on a double-log scale. The lower three solid lines are for the 2D Ising model with the lattice size $L=128,256$ and 512. The $x$ - and $y$-axis are on the bottom and right sides. According to Eq. (9), the curves collapse to the curve at $L^{\prime}=256$. Circles and triangles correspond to $L=512$ and 128 respectively. The upper three solid lines are for the 3D Ising model with $L=32,64$ and 128 . The $x$ - and $y$-axis are on the top and left sides. Data collapse is observed. Circles and triangles correspond to $L=128$ and 32 respectively. 


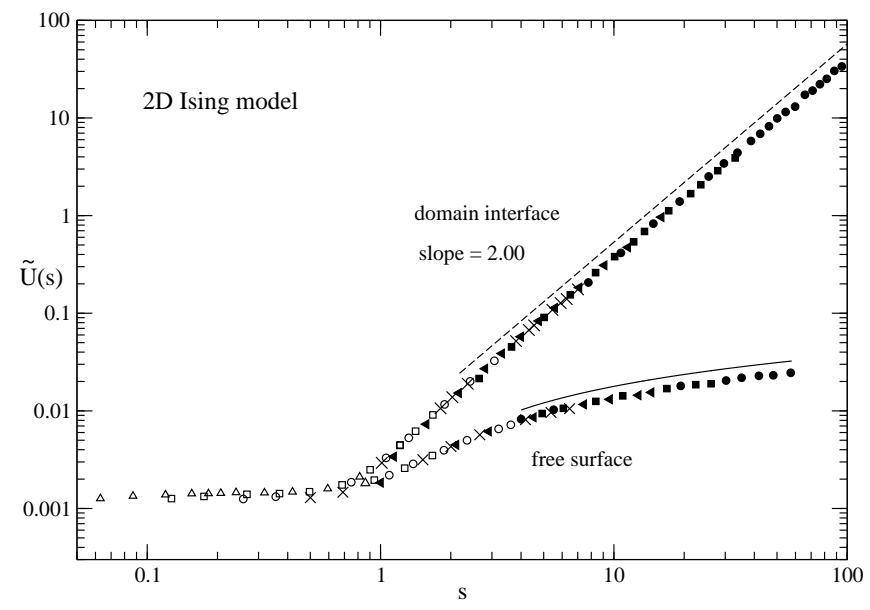

FIG. 9: The scaling function $\widetilde{U}(s)$ with $s=t^{1 / z} / x$ is plotted on a double-log scale, for the 2D Ising model with the domain interface and free surface. Data collapse for different $x$ is observed. The dashed line shows the power law fit, and the solid line indicates the logarithmic fit.

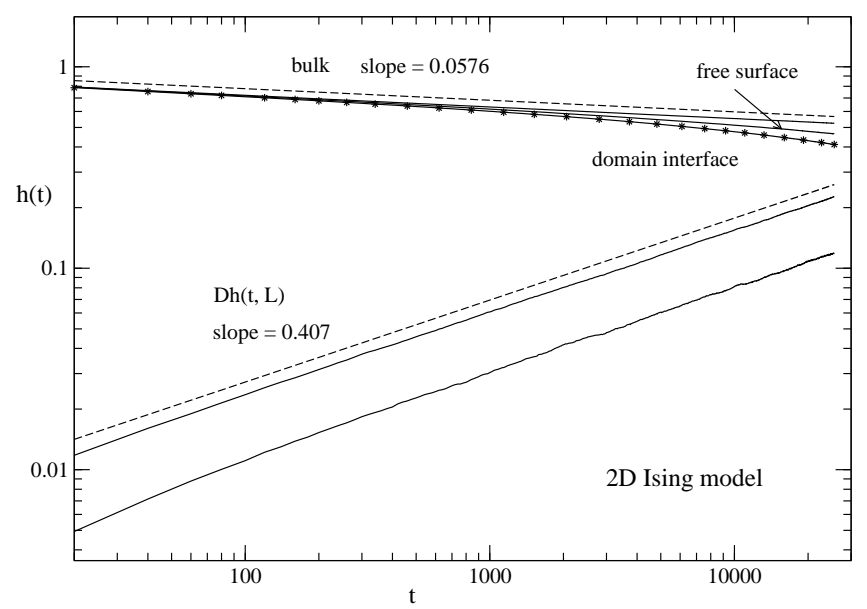

FIG. 10: The height function $h(t)$ of the 2D Ising model is plotted on a double-log scale. The three upper solid lines are for the domain interface, free surface and bulk (from below). The lower solid lines are the pure height function $D h(t, L)$ of the free surface and domain interface (from below). Dashed lines show the power-law fits, and stars are from a double power-law fit. 


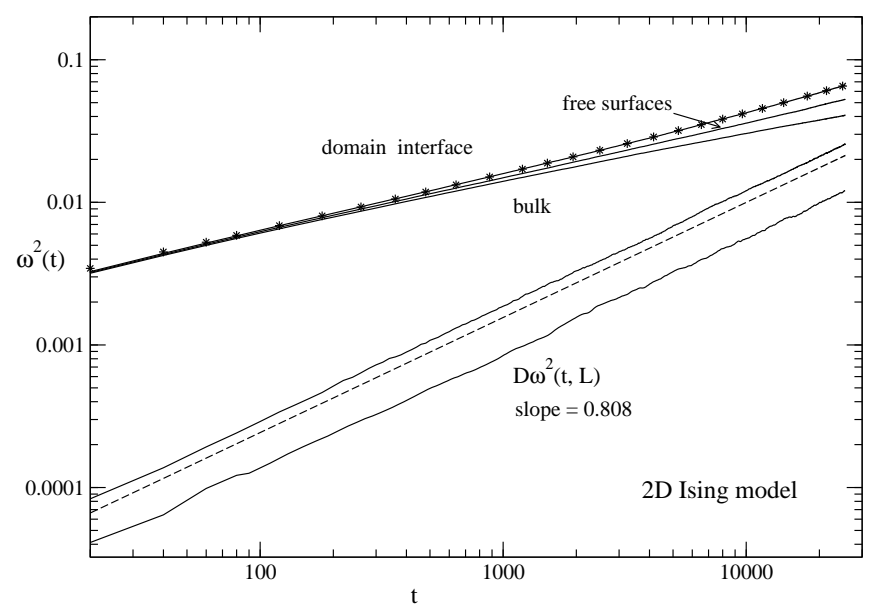

FIG. 11: The roughness function $\omega^{2}(t)$ of the 2D Ising model is plotted on a double-log scale. The three upper solid lines are for the domain interface, free surface and bulk (from above). The lower solid lines are the pure roughness function $D \omega^{2}(t, L)$ for the domain interface and free surface (from above). Dashed lines show the power-law fits, and stars are from a double power-law fit.

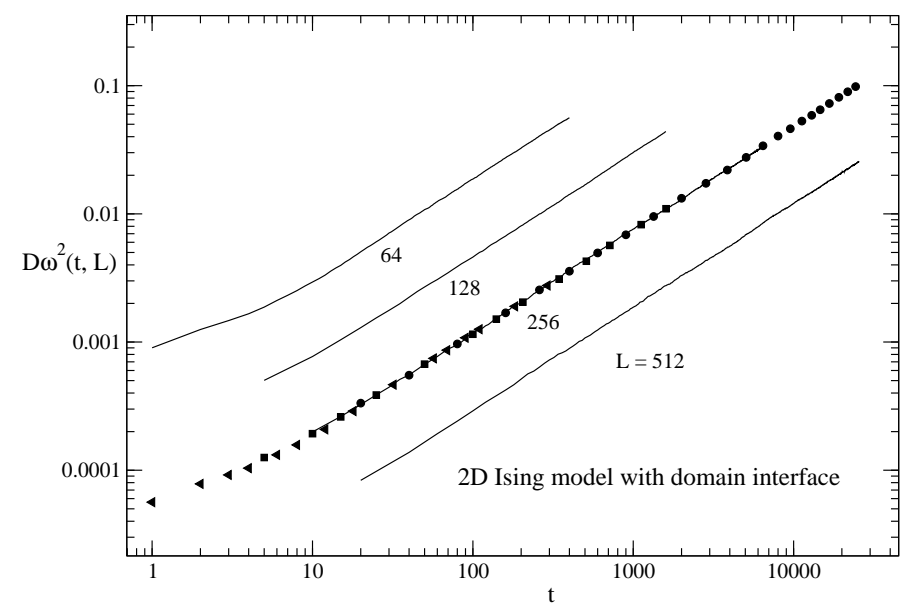

FIG. 12: The pure roughness function $D \omega^{2}(t, L)$ of the domain interface is plotted for the 2D Ising model with solid lines on a double-log scale, for $L=512,256,128$ and 64 (from below). According to Eq. (19), data collapse is observed at the curve of $L^{\prime}=256$. Solid circles, solid squares, and solid triangles correspond to $L=512,128$ and 64 respectively. 